\title{
On a long-term dynamics of the magnetised solar tachocline
}

\author{
Eun-jin Kim and N. Leprovost
}

\author{
Department of Applied Mathematics, University of Sheffield, Sheffield S3 7RH, UK \\ e-mail: e.kim@sheffield.ac.uk
}

Received 7 September 2006 / Accepted 12 January 2007

\begin{abstract}
Aims. We investigate the confinement and long-term dynamics of the magnetised solar tachocline.

Methods. Starting from first principles, we derive the values of turbulent transport coefficients in the magnetised solar tachocline and then explore the implications for the confinement and long-term dynamics of the tachocline.

Results. For reasonable parameter values, the turbulent eddy viscosity is found to be negative, with turbulence enhancing the radial shear in the tachocline. Both magnetic diffusivity and thermal diffusivity are severely quenched, with values much smaller than the magnitude of the eddy viscosity. The effect of the meridional circulation on momentum transport via the hyperviscosity becomes important when the radial shear becomes large (larger than the presently inferred value) due to negative viscosity. The results imply that the tachocline develops too strong radial shear to be a stationary Hartmann layer. In the limit of strong radiative damping where the turbulence is active on very small scales $\left(<10^{-4} R_{\odot}\right)$, the eddy viscosity can become positive although its effect is likely to be dominated by the hyperviscosity. In comparison with the momentum transport, the transport of magnetic field, heat, and passive particles is more severely quenched. The results imply that the thickness of the tachocline is of order $10^{-3} R_{\odot}-10^{-2} R_{\odot}$, independent of the strength of magnetic fields. In addition, the momentum transport is much more efficient than the particle mixing in the tachocline, consistent with the observations.
\end{abstract}

Key words. turbulence - magnetohydrodynamics (MHD) - Sun: interior - Sun: magnetic fields - Sun: rotation - waves

\section{Introduction}

A consistent theory of transport in the solar interior (in particular, the tachocline) is essential to the understanding of the evolution of solar rotation and magnetic fields and the distribution of chemical species. While in the standard solar model (Stix 1989) turbulence is assumed to be absent in the interior, observations (e.g., Pinsonneault et al. 1989) and numerical simulations (e.g., Rüdiger \& Kitchatinov 1996) suggest that transport in this region - although not so fast as turbulent transport (e.g., such as in the convection zone) - should be faster than molecular processes to be consistent with the current rotational profile and surface depletion of light elements. Such a modest transport could be due to waves via dissipative processes (e.g., radiative damping of gravity waves). Another interesting possibility, which has not received much attention, is that turbulence is present in the interior due to a variety of instabilities (e.g., see Spruit 1999, and references therein), but that the overall transport due to this turbulence is considerably reduced as a result of turbulence regulation. Our previous works (Kim 2005; Kim \& Leprovost 2007) have shown that stable stratification as well as shearing by the radial differential rotation in the tachocline can precisely do this as the excitation of gravity waves reduces the stochasticity in turbulent flow while shearing enhances the overall dissipation (see also Kim 2004).

The turbulent transport reduction can also be caused by magnetic fields (e.g., Cattaneo \& Vainshtein 1991; Gruzinov \& Diamond 1994; Kim \& Dubrulle 2001; Kim 2006). In the tachocline, a strong toroidal magnetic field of the strength $10^{4}-10^{5} \mathrm{G}$ is believed to be present, which can easily be generated when a weak poloidal magnetic field is sheared by differential rotation in the tachocline. A poloidal magnetic field here could be either of primordial origin evolving on a long evolutionary time scale (i.e., slow tachocline), or generated by dynamo process operating on a fast time scale of the solar cycle (i.e. fast tachocline) (see e.g., Gilman 2000; Petrovay 2003). Thus, magnetic fields can potentially play a crucial role in the transport of momentum, chemical species, and magnetic flux on long and/or short time scales. In particular, on an evolutionary time scale, the tachocline may be considered as a boundary layer between the uniformly rotating radiative interior and differentially rotating convection zone with latitudinal variation (Rüdiger \& Kitchatinov 1996; Gough \& McIntyre 1998; MacGregor \& Charbonneau 1999), and the dynamics of this boundary layer crucially depends on the values of the effective magnetic diffusivity, eddy viscosity, etc. The understanding of this boundary layer thus requires the prediction of these turbulent transport coefficients, derived from first principles. Furthermore, momentum transport and chemical mixing across the tachocline play a crucial role in the evolution of solar differential rotation and the distribution of chemical species. In particular, the present solar rotational profile and surface depletion of light elements (lithium) (Schatzman 1993) indicate that the angular momentum transport must have been more efficient than the particle mixing in the solar interior (e.g. Pinsonneault et al. 1989). This should be explained by a consistent theory of momentum transport and chemical mixing in the tachocline, rather than invoking a crude parameterization as has often been done by previous authors.

The purpose of this paper is to provide a consistent theory of turbulent transport in the magnetised tachocline and then investigate its implications for a long-term dynamics of the (slow) tachocline. Special attention is paid to the elucidation of different effects on transport of shearing, stable stratification, and magnetic fields, identifying what is most likely to be the main 
mechanism for turbulence regulation in the tachocline. The remainder of the paper is organized as follows. We elucidate the effects of gravity-Alfven waves on turbulent transport in Sect. 2. In Sect. 3, we incorporate the effect of shear flow given by the radial differential rotation and provide the theoretical predictions for turbulent coefficients in the stratified magnetised tachocline. We elaborate on implications of the results for a long-term dynamics of the tachocline in Sect. 4. Section 5 is devoted to the discussion of the limit of strong radiative damping. Section 6 contains the conclusions and discussions.

\section{Turbulent transport in 2D MHD with density stratification}

We envision that turbulence in the tachocline is driven externally, for instance, when plumes penetrating from the convection zone randomly stir/perturb the region. In the presence of density stratification and magnetic fields, this random stirring will excite both gravity and Alfven waves in the tachocline, which will in turn increase the memory of fluid motion which would otherwise be random and incoherent. As a result, turbulent transport is expected to be reduced (e.g. see Kim \& Leprovost 2007). In Kim \& Leprovost (2007), we have shown that stable stratification (gravity waves) leads to transport property in the three dimensional hydrodynamic (3D HD) turbulence very similar to that in the two dimensional hydrodynamic (2D HD) turbulence (without the latitudinal dependences) with a negative viscosity in both $2 \mathrm{D}$ and $3 \mathrm{D}$. We recall that without stratification the eddy viscosity is negative in $2 \mathrm{D}$ HD case while it is positive in $3 \mathrm{D}$ HD case. Magnetic fields also tend to make the property of $2 \mathrm{D}$ turbulence more like that of 3D turbulence, leading to a positive viscosity even in 2D (Kim \& Dubrulle 2001). Therefore, for simplicity, we model our stratified magnetized tachocline by a $2 \mathrm{D}$ incompressible fluid in a local cartesian coordinates $x$ and $y$. Here, $x$ and $y$ represent radial and azimuthal directions, respectively. We consider a uniform toroidal magnetic field $\boldsymbol{B}=B_{0} \hat{y}=\left(0,-\partial_{x} a_{0}, 0\right)$ and represent the differential rotation by a large-scale shear flow $\boldsymbol{U}_{0}=U_{0}(\boldsymbol{x}) \hat{y}$ parallel to the toroidal magnetic field. Note that a similar cartesian 2D model for the tachocline was also adopted in Kim \& MacGregor (2001). Furthermore, we adopt the Boussinesq approximation to capture the effect of density stratification and quasi-linear analysis by assuming that total mass density $\rho=\rho_{0}+\rho^{\prime}$, particle density for chemical species $n=n_{0}+n^{\prime}$, vorticity $\omega=\omega^{\prime}=\omega_{z}^{\prime}=\partial_{x} v_{y}-\partial_{y} v_{x}$, and magnetic potential $a=a_{0}+a^{\prime}$ consist of mean and fluctuating components, denoted by subscript ' 0 ' and prime, respectively. The governing equations for the fluctuations $\omega^{\prime}, a^{\prime}$, and $\rho_{1}=\rho^{\prime} / \bar{\rho}$ ( $\bar{\rho}$ is the mean constant mass density) can then be written as follows:

$$
\begin{aligned}
\left(\partial_{t}+U_{0} \partial_{y}\right) \omega^{\prime} & =g \partial_{y} \rho_{1}-B_{0} \partial_{y} \nabla^{2} a^{\prime}+v \nabla^{2} \omega^{\prime}+f, \\
\left(\partial_{t}+U_{0} \partial_{y}\right) a^{\prime} & =-v_{x} \partial_{x} a_{0}+\eta \nabla^{2} a^{\prime}, \\
\left(\partial_{t}+U_{0} \partial_{y}\right) \rho_{1} & =\frac{N^{2}}{g} v_{x}+\mu \nabla^{2} \rho_{1} .
\end{aligned}
$$

Here, $v, \eta$ and $\mu$ are molecular viscosity, Ohmic diffusivity and thermal diffusivity, respectively; $f$ in Eq. (1) is the small-scale forcing driving turbulence; $N=\sqrt{-g\left(\partial_{x} \rho_{0}+\bar{\rho} g / c_{\mathrm{s}}^{2}\right) / \bar{\rho}}$ is the Brunt-Väisälä frequency; $c_{\mathrm{s}}$ is the sound speed; $\rho_{0}=\rho_{0}(x)$ and $\bar{\rho}$ are the mean background and constant mass densities, respectively. Note that the typical values of $v, \mu, \eta$, and $N$ in the tachocline are $10^{2} \mathrm{~cm}^{2} \mathrm{~s}^{-1}, 10^{7} \mathrm{~cm}^{2} \mathrm{~s}^{-1}, 10^{4} \mathrm{~cm}^{2} \mathrm{~s}^{-1}$, and $3 \times 10^{-3} \mathrm{~s}^{-1}$, respectively.
To elucidate the role of magnetic fields and stable stratification in transport, it is illuminating to examine their effect on the diffusion of magnetic flux. To this end, we ignore the large-scale shear flow $U_{0}$ and forcing $f$ and recast Eqs. (1)-(3) as follows:

$$
\begin{aligned}
\partial_{t} \tilde{\omega} & =\mathrm{i} g k_{y} \tilde{\rho}_{1}+\mathrm{i} k_{y} B_{0} k^{2} \tilde{a}-v k^{2} \tilde{\omega}, \\
\partial_{t} \tilde{a} & =\mathrm{i} B_{0} k_{y} \tilde{\omega} / k^{2}-\eta k^{2} \tilde{a}, \\
\partial_{t} \tilde{\rho}_{1} & =\frac{N^{2}}{g} \frac{\mathrm{i} k_{y} \tilde{\omega}}{k^{2}}-\mu k^{2} \tilde{\rho}_{1} .
\end{aligned}
$$

Here, $\tilde{\omega}, \tilde{a}$ and $\tilde{\rho}_{1}$ are the Fourier transform of $\omega^{\prime}, a^{\prime}$ and $\rho_{1}$. Equations (5) and (6) give us $\partial_{t} \tilde{\rho}_{1} / \partial_{t} \tilde{a} \sim \tilde{\rho}_{1} / \tilde{a} \simeq N^{2} / g B_{0}$ for small dissipation (i.e. $\mu \sim \eta \sim v \sim 0$ ), which can then be used in Eq. (4) to obtain

$\partial_{t} \tilde{\omega} \simeq \mathrm{i} k_{y} B_{0} k^{2}\left[\frac{N^{2}}{k^{2} B_{0}^{2}}+1\right] \tilde{a}$.

By using Eqs. (5) and (7), we can compute the magnetic flux as

$$
\begin{aligned}
\Gamma_{x} & =\left\langle v_{x} a^{\prime}\right\rangle \\
& =\int \mathrm{d}^{2} k \mathrm{~d} t\left\langle\partial_{t} \tilde{v}_{x}(\boldsymbol{k}) \tilde{a}(-\boldsymbol{k})+\tilde{v}_{x}(\boldsymbol{k}) \partial_{t} \tilde{a}(-\boldsymbol{k})\right\rangle \\
& \simeq \frac{\tau B_{0}}{2}\left[\left\langle v^{2}\right\rangle-s\left\langle b^{2}\right\rangle\right] .
\end{aligned}
$$

Here, $\boldsymbol{b}=\nabla \times a^{\prime} \hat{z}, s=1+N^{2} / k^{2} B_{0}^{2}$, and $\int \mathrm{d} t \sim \tau$ is used where $\tau$ is the characteristic time scale of turbulence (see, e.g., Gruzinov \& Diamond 1994). For stationary fluctuations, the flux $\Gamma_{x}$ is related to magnetic energy $\left\langle b^{2}\right\rangle$ as $B_{0} \Gamma_{x}=\eta\left\langle b^{2}\right\rangle$, thereby giving us $\eta_{\mathrm{T}}=\Gamma_{x} / B_{0}$ as:

$$
\eta_{\mathrm{T}} \simeq \frac{\eta_{\mathrm{T}}^{0}}{1+s \frac{\eta_{\mathrm{T}}^{0} B_{0}^{2}}{\eta\left\langle v^{2}\right\rangle}}
$$

where $\eta_{\mathrm{T}}^{0}=\tau\left\langle v^{2}\right\rangle / 2$ is the turbulent diffusivity in the absence of magnetic field $\left(B_{0} \rightarrow 0\right)$ and stratification $(N \rightarrow 0)$. Equation (9) immediately shows that magnetic diffusivity $\eta_{\mathrm{T}}$ is severely quenched for large $R_{\mathrm{m}}=\eta_{\mathrm{T}}^{0} / \eta$ as either the magnetic field or density stratification becomes strong. This is because the excitation of waves (Alfven or gravity waves) increases the memory of turbulent eddies, reducing their stochasticity which is essential for turbulent transport. Note that Eq. (9) recovers the 2D MHD result as $N \rightarrow 0$ (e.g. Gruzinov \& Diamond 1994).

Since in the tachocline the radiative damping is much larger than ohmic diffusivity and viscosity, it is instructive to consider the limit of strong radiative damping $\mu \gg \eta \sim v$ (with $\eta \sim v \sim 0$ ) to elucidate the effect of radiative damping (recall that the result (9) is valid in the limit of small dissipation $(\mu \sim \eta \sim v \sim 0)$ ).

In this case, Eq. (6) can be approximated as $N^{2} \mathrm{i} k_{y} \tilde{\omega} / g k^{2} \sim$ $\mu k^{2} \tilde{\rho}_{1}$. A similar analysis then gives us the magnetic diffusivity

$$
\eta_{\mathrm{T}} \simeq \frac{\eta_{\mathrm{T}}^{0}}{\lambda+\frac{\eta_{\mathrm{T}}^{0} B_{0}^{2}}{\eta\left\langle v^{2}\right\rangle}}
$$

where $\lambda=1+\tau k_{y}^{2} N^{2} / \mu k^{4}$. The contribution from gravity waves to the transport reduction (the denominator of $\eta_{\mathrm{T}}$ ) in Eq. (10) is $\tau k_{y}^{2} N^{2} / \mu k^{4}$, which should be compared with $\tau N^{2} / \eta k^{2}$ in Eq. (9). The ratio of the two is thus roughly $\eta / \mu$, which becomes very small for strong radiative damping (i.e., for large $\mu$ ). That is, magnetic diffusivity in Eq. (10) is less reduced by stratification in the case of a strong radiative damping, as compared to that in the case of weak damping (Eq. (9)). This is simply because 
the radiative damping weakens buoyancy effect. In the extreme limit of $\mu \rightarrow \infty$, the effect of stratification disappears with $\lambda \rightarrow 1$ in Eq. (10), recovering the 2D MHD result in the absence of stratification.

\section{Consistent theory with the radial differential rotation}

In Sect. 2, we have shown that both stable stratification and magnetic fields can severely quench the transport of magnetic field. In this section, we present a consistent theory of turbulent transport in the stratified magnetised tachocline, by incorporating a background shear flow $U_{0} \hat{y}$ provided by the radial differential rotation. The latitudinal differential rotation is neglected compared to the radial differential rotation since it is weaker in the tachocline due to thin tachocline (of thickness $<0.03-0.05$ of the solar radius) (see Leprovost \& Kim 2006, for the dynamics and the effect of latitudinal differential rotation). This shear flow plays a crucial role in regulating turbulence and turbulent transport by shearing (e.g., see Kim 2005). For simplicity, we assume a linear shear flow $U_{0} \hat{y}=-x \mathcal{A} \hat{y}$, where $\mathcal{A}=\left|\partial_{x} U_{0}\right|>0$ is the radial shear (or shearing rate), which is assumed to be positive without loss of generality. In order to incorporate the shearing effect non-perturbatively, we employ the special Fourier transform for the fluctuating quantities $\phi^{\prime}$ :

$\phi^{\prime}(\boldsymbol{x}, t)=\frac{1}{(2 \pi)^{3}} \int \mathrm{d}^{3} k \tilde{\phi}(\boldsymbol{k}, t) \exp \left\{\mathrm{i}\left(k_{x}(t) x+k_{y} y+k_{z} z\right)\right\}$,

where $k_{x}(t)$ linearly increases in time due to shearing as

$k_{x}(t)=k_{x}(0)+k_{y} \mathcal{A} t$.

Here, $\mathcal{A}=\left|\partial_{x} U_{0}\right|$ is the shearing rate of the shear flow. In this section, we consider the case where the radiative damping rate is small compared to the shearing rate. That is, we consider a strong shear limit where $\xi_{\mu}=\mu k_{y}^{2} / \mathcal{A} \ll 1$. Here, $l_{\mathrm{f}}=1 / k_{y}$ is the characteristic length scale of the forcing. Thus, $\xi_{\mu}(\ll 1)$ is a small parameter, representing the strong shear limit. For typical solar parameter values $\mu \sim 10^{7} \mathrm{~cm}^{2} \mathrm{~s}^{-1}$ and $\mathcal{A} \sim 3 \times 10^{-6} \mathrm{~s}^{-1}$, this is a good assumption valid for a broad range of characteristic length scales of the forcing $l_{\mathrm{f}}=1 / k_{y} \gtrsim 10^{6}-10^{7} \mathrm{~cm}\left(\sim 10^{-4} R_{\odot}\right.$ where $R_{\odot} \sim 5 \times 10^{10} \mathrm{~cm}$ is the solar radius). Note that the opposite limit of strong radiative damping is considered in Sect. 5.

For $\xi_{\mu} \ll 1$, the coupled Eqs. (1)-(3) can easily be combined to form the following equation for $\hat{a}=$ $\tilde{a} \exp \left[\mu\left(k_{x}^{3} / 3 k_{y} \mathcal{A}+k_{y}^{2} t\right) / 2\right]$ as

$\partial_{\tau}\left[\left(1+\tau^{2}\right) \partial_{\tau} \hat{a}\right]+\gamma^{2}\left[\left(1+\tau^{2}\right)+\beta\right] \hat{a} \simeq \frac{\mathrm{i} B_{0}}{\mathcal{A}^{2} k_{y}} \hat{f}(\tau)$,

to leading order in $\xi_{\mu}$. Here, $\tau=k_{x} / k_{y}=k_{x}(0) / k_{y}+\mathcal{A} t$, $\gamma=\left|B_{0} k_{y} / \mathcal{A}\right|=\left|\omega_{\mathrm{A}} / N\right|, \beta=N^{2} / B_{0}^{2} k_{y}^{2}=N^{2} / \omega_{\mathrm{A}}^{2}, \omega_{\mathrm{A}}=$ $\left|B_{0} k_{y}\right|$ is the Alfven frequency of the mode $k_{y}$, and $\hat{f}=$ $\tilde{f} \exp \left[\mu\left(k_{x}^{3} / 3 k_{y} \mathcal{A}+k_{y}^{2} t\right) / 2\right]$.

The solution to Eq. (13) in the limit of strong magnetic field $\gamma \gg 1$ and stratification $N^{2} / \mathcal{A}^{2} \gg 1$ can be found as

$\hat{a}(\tau) \simeq$

$\frac{\mathrm{i} B_{0}}{\mathcal{A}^{2} k_{y} \gamma} \int \mathrm{d} \tau_{1} \frac{\sin \gamma\left[Q(\tau)-Q\left(\tau_{1}\right)\right] \hat{f}\left(\tau_{1}\right)}{\left[\left(1+\tau^{2}\right)\left(1+\tau_{1}^{2}\right)\left(1+\tau^{2}+\beta\right)\left(1+\tau_{1}^{2}+\beta\right)\right]^{1 / 4}}$,

where $Q(\tau)=\int^{\tau} \mathrm{d} \tau_{1} \sqrt{\frac{1+\tau_{1}^{2}+\beta}{1+\tau_{1}^{2}}}$. Note that the strong magnetic field limit $\gamma>1$ holds for a broad range of length scales of the forcing $l_{\mathrm{f}} \lesssim L_{B}=10^{10} \mathrm{~cm}$ for $B_{0}=10^{4}-10^{5} \mathrm{G}$ in the tachocline. To obtain the turbulent coefficients, we assume that the forcing is homogeneous with a short correlation time $\tau_{\mathrm{f}}$;

$\left\langle\tilde{f}\left(\boldsymbol{k}_{1}, t_{1}\right) \tilde{f}\left(\boldsymbol{k}_{2}, t_{2}\right)\right\rangle=\tau_{\mathrm{f}}(2 \pi)^{2} \delta\left(t_{1}-t_{2}\right) \delta\left(\boldsymbol{k}_{1}+\boldsymbol{k}_{2}\right) \phi\left(\boldsymbol{k}_{2}\right)$.

By using Eqs. (1)-(7), (14) and (15), a long but straightforward algebra then gives us the eddy viscosity defined by $\left\langle v_{x} v_{y}-\right.$ $\left.b_{x} b_{y}\right\rangle=-v_{\mathrm{T}} \partial_{x} U_{0}=v_{\mathrm{T}} \mathcal{A}\left(\boldsymbol{b}=\nabla \times a^{\prime} \hat{z}\right)$ :

$$
\begin{aligned}
v_{\mathrm{T}} & =\frac{\tau_{\mathrm{f}}}{2 \mathcal{A}^{2}} \int \frac{\mathrm{d}^{2} k}{(2 \pi)^{2}} \frac{\phi(\boldsymbol{k})}{k_{y}^{2}}\left[-1+\frac{1}{\sqrt{1+\beta}}\right] \\
& \sim \frac{1}{\mathcal{A}^{2}}\left[-1+\frac{1}{\sqrt{1+\beta}}\right] \mathcal{F} .
\end{aligned}
$$

Here, $\phi(\boldsymbol{k})$ is the power spectrum of the forcing defined in Eq. (15); $\mathcal{F} \sim \int \mathrm{d}^{2} k \phi(\boldsymbol{k}) / k_{y}^{2} \sim v_{\mathrm{f}}^{2} / \tau_{\mathrm{f}}$ is the strength of the forcing with the characteristic velocity $v_{\mathrm{f}}$ and correlation time $\tau_{\mathrm{f}}$; $\beta=N^{2} / B_{0}^{2} k_{y}^{2}=N^{2} / \omega_{\mathrm{A}}^{2}, \omega_{\mathrm{A}}=\left|B_{0} k_{y}\right| ; \mathcal{A}=\left|\partial_{x} U_{0}\right|$ is the radial shear. Interestingly, the eddy viscosity in Eq. (16) is always negative regardless of the relative strength of stratification to magnetic field (i.e., $\beta=N^{2} / \omega_{\mathrm{A}}^{2}$ ). In 2D MHD, the Maxwell stress exactly cancels the Reynolds stress to leading order while the incomplete cancellation between the two in the next order gives a small positive eddy viscosity $\left(\propto 1 / B_{0}^{2}\right)$ (Kim \& Dubrulle 2001). The cancellation of these leading order contributions in $2 \mathrm{D}$ unstratified MHD can easily be checked in Eq. (16) by putting $\beta=0$, which gives $\nu_{\mathrm{T}}=0$. In contrast, in a stratified medium with $\beta \neq 0$, the leading order cancellation in Eq. (16) is not perfect, giving a net negative eddy viscosity. This can be shown to be due to the fact that the exact equipartition between the kinetic energy and magnetic energy (for pure Alfven waves) is broken by buoyancy. A negative eddy viscosity, in a sharp contrast to a positive eddy viscosity, signifies an anti-diffusive momentum transport against the gradient of the shear flow. In other words, the overall momentum transport due to turbulence accelerates the mean flow, accentuating its gradient rather than eradicating it. A similar tendency of the acceleration of the mean flow in the magnetised tachocline can also be due to the direct momentum deposition of gravity waves via radiative damping (Kim \& MacGregor 2001).

For clarity, we now examine the behavior of $v_{\mathrm{T}}$ as a function of $\beta$. For large $\beta$ (strong stratification/weak magnetic field), $v_{\mathrm{T}} \propto\left[-1+\left|B_{0} k_{y} / N\right|\right] / \mathcal{A}^{2}$, showing that the effect of magnetic fields tends to make eddy viscosity positive. In the opposite limit of small $\beta$ (weak stratification/strong magnetic field), $\nu_{\mathrm{T}} \propto-\beta / 2 \mathcal{A}^{2}=-N^{2} / 2 B_{0}^{2} \mathcal{A}^{2}$, whose absolute magnitude $\left|\nu_{\mathrm{T}}\right|$ is small compared to the 2D HD case where $v_{\mathrm{T}} \propto-1 / \mathcal{A}^{2}$. This again reflects the tendency of magnetic fields making the eddy viscosity less negative (i.e. more positive). Note that for parameter values typical of the tachocline, the cross-over scale $L_{N}$ from $\beta>1$ to $\beta<1$ is roughly $L_{N}=10^{7}-10^{8} \mathrm{~cm}$.

To recapitulate, the result (16) shows a tendency of a negative eddy viscosity in a stratified medium despite the presence of a rather strong magnetic field. Thus, the turbulent transport in the tachocline would amplify the shear provided by the radial differential rotation for reasonable values of parameters. We emphasize that the negative eddy viscosity represents the amplification of a large-scale shear flow at the expense of small-scale turbulence. Note that if the scale of the mean flow continues to decrease and becomes comparable to the characteristic scale of small-scale (due to the negative viscosity), the concept of eddy viscosity becomes invalid. 
The value of magnetic diffusivity, defined by $\left\langle a^{\prime} v_{x}\right\rangle=$ $-\eta_{\mathrm{T}} \partial_{x} a_{0}$, depends on whether $\alpha=\left(\xi_{\mu} / 3\right)(1+\beta)^{3 / 2}$ is larger or smaller than unity. First, in the case $\alpha \ll 1$, which is valid on scales $L<L_{M}=B_{0}^{3} \mathcal{A} / \mu N^{3} \sim 10^{8} \mathrm{~cm}\left(10^{11} \mathrm{~cm}\right)$ for $B_{0} \sim 10^{4} \mathrm{G}$ $\left(10^{5} \mathrm{G}\right)$ for $\beta>1$ or on scales $L>10^{6} \sim 10^{7} \mathrm{~cm}\left(\right.$ e.g. $\left.\xi_{\mu} \ll 1\right)$ for $\beta>1$, we can obtain

$$
\begin{aligned}
\eta_{\mathrm{T}} & =\frac{\tau_{\mathrm{f}}}{2 B_{0}^{2}} \int \frac{\mathrm{d}^{2} k}{(2 \pi)^{2}} \frac{\phi(\boldsymbol{k})}{k_{y}^{4}} \xi_{\eta} G_{0} \frac{1}{\sqrt{1+\beta}} \\
& \sim \frac{1}{B_{0}^{2} k_{y}^{2}} \xi_{\eta}^{2 / 3}\left(\frac{\eta}{\mu}\right)^{1 / 3} \frac{1}{\sqrt{1+\beta}} \mathcal{F} .
\end{aligned}
$$

Here, $G_{0}=\left(3 / \xi_{\mu}\right)^{1 / 3} \Gamma(1 / 3) / 3 ; \xi_{\mu}=\mu k_{y}^{2} / \mathcal{A} \ll 1 ; \xi_{\eta}=$ $\eta k_{y}^{2} / \mathcal{A} \ll 1 ; \Gamma(x)$ is the Gamma function; $\mathcal{F} \sim \int \mathrm{d}^{2} k \phi(\boldsymbol{k}) / k_{y}^{2} \sim$ $v_{\mathrm{f}}^{2} / \tau_{\mathrm{f}}$ is again the strength of the forcing with the characteristic velocity $v_{\mathrm{f}}$ and correlation time $\tau_{\mathrm{f}}$. In comparison, for $(\beta \gg)$ $\alpha \gg 1$, we obtain

$$
\begin{aligned}
\eta_{T} & =\frac{\tau_{\mathrm{f}}}{2 N^{2}} \int \frac{\mathrm{d}^{2} k}{(2 \pi)^{2}} \frac{\phi(\boldsymbol{k})}{k_{y}^{2}} \xi_{\eta} G_{1} \\
& \sim \frac{1}{N^{2}} \xi_{\eta}^{1 / 3}\left(\frac{\eta}{\mu}\right)^{2 / 3} \mathcal{F},
\end{aligned}
$$

where $G_{1}=\left(3 / \xi_{\mu}\right)^{2 / 3} \Gamma(2 / 3) / 3$. In each case, the density (heat) diffusivity $\mu_{\mathrm{T}}\left(\left\langle\rho^{\prime} v_{x}\right\rangle=-\mu_{\mathrm{T}} \partial_{x} \rho_{0}\right)$ is given by

$\mu_{\mathrm{T}} \simeq \frac{\mu}{\eta} \eta_{\mathrm{T}}$

\section{(See also Kim 2006.)}

Equation (17) shows that the turbulent diffusion of magnetic field $\left(\eta_{\mathrm{T}}\right)$ can severely be quenched by a strong mean magnetic field and stratification, proportional to $1 / B_{0}^{2}$ for $\beta<1$ and to $1 / B_{0} N$ for $\beta>1$, respectively. We note that $\eta_{\mathrm{T}} \propto 1 / B_{0}^{2}$ was observed in numerical simulation (e.g. Cattaneo \& Vainshtein $1991)$ of $2 \mathrm{D}$ unstratified MHD turbulence $(\beta=0)$. As the stratification becomes stronger for a fixed $B_{0}$ with a further increase in $\beta$, the diffusion is now reduced as $1 / N^{2}$ [see Eq. (18)]. It is important to note that in all cases, magnetic diffusivity has a much smaller magnitude than the eddy viscosity [in Eq. (16)], with a small value of $\eta_{\mathrm{T}} /\left|v_{\mathrm{T}}\right|$. A similar tendency was also found in the stably stratified shear turbulence without magnetic fields (Kim $\&$ Leprovost 2007). Specifically, in the strong magnetic field and weak stratification region with $\alpha<1$ and $\beta>1, \eta_{\mathrm{T}} /\left|\nu_{\mathrm{T}}\right| \sim$ $\xi_{\eta}^{2 / 3}(\eta / \mu)^{1 / 3} \mathcal{A}^{2} / N \omega_{\mathrm{A}} \ll 1$. Furthermore, the heat diffusivity $\mu_{\mathrm{T}}$ in Eq. (19), although larger than $\eta_{\mathrm{T}}$ by a factor of $\mu / \eta=10^{3}$, is yet much smaller than the magnitude of $\nu_{\mathrm{T}}$. For instance, for $\alpha<1$ and $\beta>1$, this ratio becomes $\mu_{\mathrm{T}} /\left|\nu_{\mathrm{T}}\right| \sim \xi_{\mu}^{2 / 3} \mathcal{A}^{2} / N \omega_{\mathrm{A}} \ll 1$ for typical parameter values. These results have very interesting implications for a long-term dynamics of the tachocline, as discussed in Sect. 4.

\section{Implications for a long-term dynamics of the tachocline}

The results of the present and previous paper (Kim \& Leprovost 2007; Kim \& MacGregor 2001) suggest that the uniform rotation in the radiative interior is very unlikely to be explained by hydrodynamical means as the momentum transport in stratified medium accelerates the mean flow, sharpening the gradient of radial differential rotation that has been created during the solar spin-down (see, however, Charbonnel \& Talon 2005). Previous authors (Rüdiger \& Kitchatinov 1996; Gough \& McIntyre 1998; MacGregor \& Charbonneau 1999), have however shown that a rather weak poloidal magnetic field in the radiative interior can eliminate the differential rotation, thereby leading to a uniform rotation therein. In this case, the tachocline can be envisioned as a boundary layer where the generation of the toroidal magnetic field by the shearing of the poloidal magnetic field (due to differential rotation) is balanced by the diffusion of the toroidal magnetic field while the dissipation of the radial differential rotation is balanced by the azimuthal Lorentz force associated with the large-scale toroidal and poloidal magnetic fields, i.e., Hartmann layer (Rüdiger \& Kitchatinov 1996; Gough \& McIntyre 1998; MacGregor \& Charbonneau 1999). By adopting the molecular values for viscosity, magnetic diffusivity, and heat diffusivity in the tachocline, these previous authors obtained estimates of the tachocline thickness and the strength of the interior poloidal magnetic field. However, in the case of the tachocline with residual turbulence, for instance, driven externally (e.g. plume penetration) or internally (e.g. via instability), the values of turbulent transport coefficients, instead of molecular values, should be used in the analysis of the Hartmann layer.

For the clarity of the discussion, it is worth recalling that the Hartmann layer is based on the configuration where a poloidal magnetic field is fully contained in the interior, without penetrating into the convection zone above so that the latitudinal differential rotation in the convection zone does not leave its footprint into the radiative interior. By representing the latitudinal coordinate by $z$, and by denoting the poloidal and the toroidal magnetic fields by $B_{z}$ and $B_{y}=B_{0}$, respectively, the major force balance for the toroidal magnetic field in the tachocline $B_{y}$ and the mean shear flow $U_{y}$ due to the differential rotation can roughly be expressed as

$\begin{aligned} \partial_{t} U_{y} & \sim B_{z} \partial_{z} B_{y}+v_{\mathrm{T}} \partial_{x x} U_{y}-\lambda_{\mathrm{T}} \partial_{x x x x} U_{y} \\ \partial_{t} B_{y} & \sim B_{z} \partial_{z} U_{y}+\eta_{\mathrm{T}} \partial_{x x} B_{y}\end{aligned}$

Here, $\lambda_{\mathrm{T}} \sim \mu_{\mathrm{T}}(\Omega / N)^{2}\left(R_{\odot} / \Lambda\right)^{2}$ is the hyperviscosity due to the meridional circulation (see, e.g., Spiegel \& Zahn 1992); $\Omega \sim$ $3 \times 10^{-6} \mathrm{~s}^{-1}$ and $R_{\odot} \sim 5 \times 10^{10} \mathrm{~cm}$ are the average rotation rate and solar radius, respectively; $\Lambda$ is a constant of order unity. Similarly to a positive eddy viscosity, a (positive) hyperviscosity acts to smooth out the gradient of (or damp) a large-scale shear flow.

While the values of turbulent transport $\left(\nu_{\mathrm{T}}, \eta_{\mathrm{T}}\right.$, and $\left.\lambda_{\mathrm{T}}\right)$ for the coupled system (20)-(21) have conventionally been assumed to be positive, the results in Sect. 3 show that the turbulent momentum transport in the sheared stratified turbulent tachocline is anti-diffusive with negative viscosity (i.e., $v_{\mathrm{T}}<0$ ), accelerating the mean shear flow $U_{y}$ (i.e., radial differential rotation). The negative viscosity will amplifies the shear in the differential rotation and consequently toroidal magnetic field since they are coupled via the Lorentz force (via $B_{z}$ ). The crucial question is then how the Hartmann layer is maintained with negative viscosity which tends to become unstable due to $v_{\mathrm{T}}<0$. Obviously, if the magnetic diffusivity $\eta_{\mathrm{T}}$ is large enough to overcome an unstable situation caused by the negative viscosity, the coupled system can find a stable stationary configuration. However, this is very unlikely since the magnetic diffusion is pathetically small compared to the magnitude of the eddy viscosity, as discussed in Sect. 3. Could the hyperviscosity $\lambda_{\mathrm{T}} \sim\left(R_{\odot} / \Lambda\right)^{2}(\Omega / N)^{2} \mu_{\mathrm{T}}$ due to the meridional circulation then stabilize the system? To answer this question, we note that the contribution from the hyperviscosity to Eq. (20) is of or$\operatorname{der} \lambda_{\mathrm{T}} / h^{4}$ while the contribution from the eddy viscosity is of 
order $v_{\mathrm{T}} / h^{2}$, where $h$ is of order of the tachocline thickness. Thus, the ratio of the two is roughly $\left(\mu_{\mathrm{T}} / \nu_{\mathrm{T}}\right)\left(R_{\odot} / h\right)^{2}(\Omega / N)^{2}(\mathrm{e} . \mathrm{g}$. $\sim\left(\mu k_{y}^{2} / \mathcal{A}\right)^{2 / 3}\left(\mathcal{A}^{2} / N \omega_{\mathrm{A}}\right)(\Omega / N)^{2}\left(R_{\odot} / h\right)^{2}$ for $\alpha<1$ and $\left.\beta>1\right)$, which can be shown to be small for the parameter values typical of the present solar tachocline. However, it is important to realize that this ratio $\left(\propto \mathcal{A}^{4 / 3}\right.$ for $\alpha<1$ and $\beta>1$, for instance) becomes large as the shear $\mathcal{A}$ increases. Therefore, it is plausible that as the shear is amplified via the negative eddy viscosity, the effect of the hyperviscosity becomes important and eventually dominates the negative viscosity, possibly stabilizing the system. In order for this to be the case, the shear $\mathcal{A}$ in the radial differential rotation however has to be larger than what is observed today. This can be checked by requiring $\lambda_{\mathrm{T}} / h^{2} \nu_{\mathrm{T}}>1$. For instance, in the case $\alpha<1$ and $\beta>1$, this demands $\mathcal{A} / N>$ $\left(N / \mu k_{y}^{2}\right)^{1 / 2}\left(B_{0} k_{y} / N\right)^{3 / 4}\left(h N / R_{\odot} \Omega\right)^{3 / 2}$, where $l_{\mathrm{f}}=1 / k_{y}$ is the characteristic scale of the forcing. Even if we take $B_{0} \sim 10^{4} \mathrm{G}$ and a thin tachocline $h \sim 10^{-3} R_{\odot}, \mathcal{A}>0.1 N$ for $k_{y} \sim 10^{-7} \mathrm{~cm}^{-1}$, and $\mathcal{A}>0.01 N$ for $k_{y} \sim 10^{-6} \mathrm{~cm}^{-1}$, which seem to be rather too large to be reasonable (recall $N \sim 10^{3} \mathrm{~s}^{-1}$ and $\mathcal{A} \sim 3 \times 10^{-6} \mathrm{~s}^{-1}$ are the presently inferred values in the tachocline).

To make this argument more concrete, it is instructive to examine the behavior of Eqs. (20)-(21) in more detail. To this end, we average them over the space $(\mathbf{x})$ with the approximation $\partial_{z} \sim \mathrm{i} / R_{\odot}$ and $\partial_{x} \sim 1 / h$ and use the values of $\nu_{\mathrm{T}}, \eta_{\mathrm{T}}$ and $\mu_{\mathrm{T}}$ obtained for $\alpha<1$ in Sect. 3. Here, $h$ is again the tachocline thickness. The resulting envelop equations for $\mathcal{A} \sim U_{y} / R_{\odot}$ and $B_{y}$, in a properly non-dimensonalized form, are as follows:

$\partial_{t} S=\mathrm{i} M+[\bar{v}(S, M)-\kappa \bar{\eta}(S, M)] S$,

$\partial_{t} M=\mathrm{i} S-\bar{\eta}(S, M) M$.

Here, $S=\mathcal{A} / \Omega, M=B_{y} / \Omega R_{\odot}, \bar{v}=\sigma\left[1-1 / \sqrt{1+1 / M^{2}}\right] /|S|^{2}$, and $\bar{\eta}=1 / \sqrt{M^{4}+M^{2}}|S|^{2 / 3}$. The parameter $\sigma$ appearing in $\bar{v}$ is a rough measure of $\left|v_{\mathrm{T}} / \eta_{\mathrm{T}}\right|$, which was shown to be very large in Sect. 3 because the magnetic diffusion is much smaller than the magnitude of the eddy viscosity. Specifically, $\sigma \sim$ $(\mu / \eta)^{1 / 3}\left(\mathcal{A} / \eta k_{y}^{2}\right)^{2 / 3} N^{2} / \mathcal{A}^{2} \gtrsim O\left(10^{8}\right)$ obtained by using the reasonable parameter values in the tachocline $\mathcal{A} \sim 3 \times 10^{-6} \mathrm{~s}^{-1}$, $N \sim 3 \times 10^{-3} \mathrm{~s}^{-1}$, and $k_{y} \sim 10^{-8} \mathrm{~cm}^{-1}$. The parameter $\kappa$ in Eq. (22) represents a rough measure of the ratio of the effect of hyperviscosity to that of magnetic diffusivity, with the value $\kappa \sim\left(R_{\odot} \Omega / h N\right)^{2}(\mu / \eta) \sim \lesssim O\left(10^{3}\right)$ for $h / R_{\odot}>10^{-3}$.

We note that the values of $\eta_{\mathrm{T}}, \nu_{\mathrm{T}}$ and $\mu_{\mathrm{T}}$ are all proportional to the intensity of the forcing $\mathcal{F}$, which is a free parameter in our problem. In our non-dimensionlization, this value is fixed by utilizing the observational evidence that the particle diffusivity of light elements (lithium) is about $D_{\mathrm{T}} \sim 10^{3} \mathrm{~cm}^{2} \mathrm{~s}^{-1}$ to be consistent with the present solar surface lithium depletion (e.g., see Barnes et al. 1999). Since $\eta_{\mathrm{T}} \sim(\eta / D) D_{\mathrm{T}}$ where $D \sim 20 \mathrm{~cm}^{2} \mathrm{~s}^{-1}$ and $\eta \sim 10^{4} \mathrm{~cm}^{2} \mathrm{~s}^{-1}$, we impose the condition that $\eta_{\mathrm{T}} \sim 10^{5} \mathrm{~cm}^{2} \mathrm{~s}^{-1}$ for the parameter values typical of the present sun (i.e. $B_{y} \sim 10^{5} \mathrm{G}, \mathcal{A} \sim 3 \times 10^{-6} \mathrm{~s}^{-1}$, etc). (Note that $\eta_{\mathrm{T}}$ in Eqs. (17)-(18) depends on both $\mathcal{A}$ and $B_{y}$, which change in time according to Eq. (22).)

The results from the numerical simulation of Eq. (22) for $\sigma=10^{8}$ are plotted by the cross symbols in Fig. 1, which shows $|S|=|\mathcal{A} / \Omega|$ for various values of $\kappa$. The results nicely show that the amplitude of $S$ decreases as $\kappa$ (hyperviscosity) becomes large, as expected. However, for small $\kappa$ (hyperviscosity), an amplitude of $\mathcal{A}>10^{3} \Omega$ is too large to be reasonable. For this large value of $\mathcal{A}$, which is comparable to the Brunt-Väisälä frequency $N$, the shear flow is likely to be unstable against a shear instability (see, e.g., Drazin 1981). As $\kappa$ increases, the amplitude of

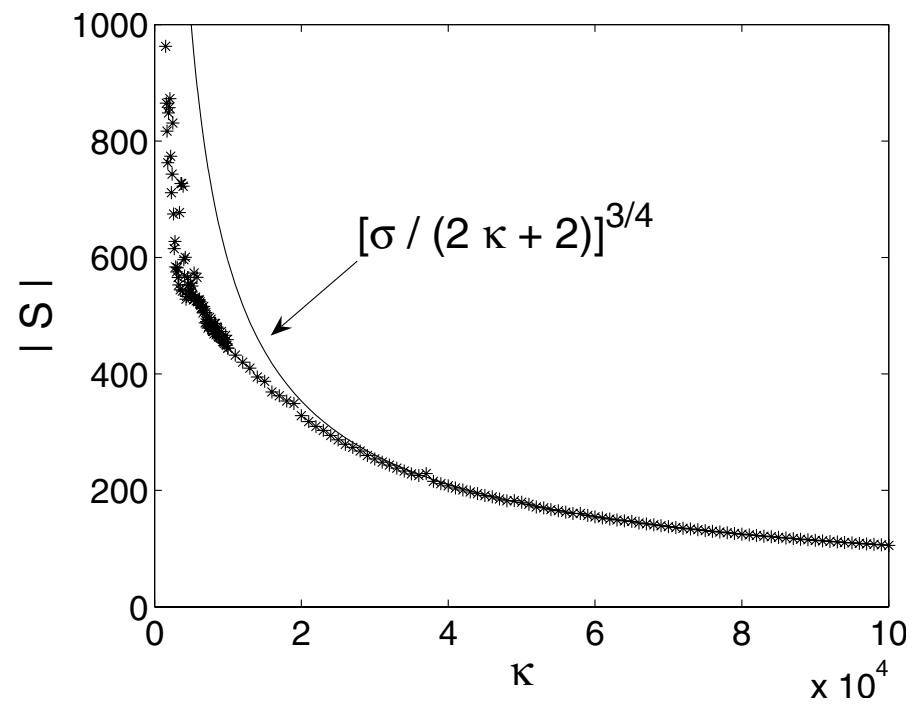

Fig. 1. The plot of the amplitude of $S=\mathcal{A} / \Omega$ as a function of $\kappa(\propto$ hyperviscosity) for $\sigma=10^{8}$. The cross symbols represent the results of the numerical simulation of the toy model Eq. (22). The dashed line denotes the asymptotic behavior, given by $[\sigma /(2 \kappa+2)]^{3 / 4}$.

$\mathcal{A}$ decreases and approaches the asymptotic value given by the dashed line $[\sigma /(2 \kappa+2)]^{3 / 4}$, which is obtained by using $|S|=|M|$. The asymptotic value indicates that $\mathcal{A} \sim 100 \Omega$ for $\kappa=10^{5}$ while $\mathcal{A} \sim 3 \Omega$ for $\kappa=10^{7}$. By using the definition of $\kappa$, one can easily show that a reasonable value of shear $\mathcal{A} \sim \Omega$ is possible only for an extremely thin tachocline with $h / R_{\odot} \sim 10^{-5}$. Since $|S|=|M|$ along the dashed line in Fig. 1, the toroidal magnetic field also seems too strong $\left(>10^{5} \mathrm{G}\right)$ to be stable (e.g. against the magnetic buoyancy instability). To summarize, the results from our toy model (22) clearly demonstrate that for reasonable parameter values, a stable stationary Hartmann layer is very unlikely in the tachocline. Thus, a large-scale shear flow is likely to become time-dependent (similarly to the behaviour found in Kim \& MacGregor 2001), or to develop a secondary instability.

\section{In the limit of the strong radiative damping}

The anti-diffusive momentum transport discussed in the previous sections originates from the stable stratification in the tachocline. The buoyancy force can however be weakened by a strong radiative damping, as demonstrated in Sect. 2. Thus, it is conceivable that if a radiative damping is large enough to weaken the buoyancy force sufficiently, the momentum transport may become diffusive, smoothing out the gradient of the radial differential rotation. In this section, we explore this possibility by considering the limit of a strong radiative damping where the density fluctuation $\rho_{1}$ in Eq. (3) is stationary with $\left(\partial_{t}+U_{0} \partial_{y}\right) \rho_{1}=0$. In order for this limit to be valid, the parameter $\xi_{\mu}=\mu k_{y}^{2} / \mathcal{A}$, which has been assumed to be small in the previous sections, is no longer small. We are however still interested in the case where the dissipation rate of magnetic field is small compared to the shearing, namely, for $\xi_{\eta}=\eta k_{y}^{2} / \mathcal{A} \ll 1$. Note that this is a valid assumption for the reasonable values of the characteristic scale of the forcing $l_{\mathrm{f}}=1 / k_{y}>10^{5} \mathrm{~cm}$ in the tachocline.

By using $N^{2} v_{x} / g+\mu \nabla^{2} \rho_{1}=0$, the coupled Eqs. (1)-(2) can easily be combined to yield

$\left[\partial_{\tau}+\frac{\bar{N}^{2}}{\left(1+\tau^{2}\right)^{2}}\right]\left[\left(1+\tau^{2}\right) \partial_{\tau} \hat{a}\right]+\gamma^{2}\left(1+\tau^{2}\right) \hat{a} \simeq \frac{\mathrm{i} B_{0}}{\mathcal{A}^{2} k_{y}} \hat{f}(\tau)$ 
to leading order in $\xi_{\eta} \ll 1$. Here, $\hat{a}=$ $\tilde{a} \exp \left[\eta\left(k_{x}^{3} / 3 k_{y} \mathcal{A}+k_{y}^{2} t\right) / 2\right] ; \hat{f}=\tilde{f} \exp \left[\eta\left(k_{x}^{3} / 3 k_{y} \mathcal{A}+k_{y}^{2} t\right) / 2\right] ;$ $\tau=k_{x} / k_{y}=k_{x}(0) / k_{y}+\mathcal{A} t, \gamma=\left|B_{0} k_{y} / \mathcal{A}\right|, \omega_{\mathrm{A}}=\left|B_{0} k_{y}\right|$, and $\bar{N}^{2}=N^{2} / \mathcal{A}\left(\mu k_{y}^{2}\right)$. The solution to Eq. (23) can be obtained in the limit $\xi_{\eta}<1$ :

$\hat{a}(\tau) \simeq \frac{\mathrm{i} B_{0}}{\mathcal{A}^{2} k_{y} \gamma} \int \mathrm{d} \tau_{1} \frac{\sin \gamma\left[\varphi(\tau)-\varphi\left(\tau_{1}\right)\right] \hat{f}\left(\tau_{1}\right) \mathrm{e}^{-\bar{N}^{2}\left(\varpi(\tau)-\varpi\left(\tau_{1}\right)\right.}}{\left(1+\tau^{2}\right)^{1 / 2}\left(1+\tau_{1}^{2}\right)^{1 / 2} \psi\left(\tau_{1}\right)}$.

Here, $\varphi(\tau)=\int^{\tau} \mathrm{d} \tau_{1} \psi\left(\tau_{1}\right), \psi(\tau)=1-\left[\left(1+\tau^{2}\right)^{-2}-\bar{N}^{2} \tau(1+\right.$ $\left.\left.\tau^{2}\right)^{-3}+\bar{N}^{4}\left(1+\tau^{2}\right)^{-4} / 4\right] / 2 \gamma^{2}$, and $\varpi(\tau)=\left[\tan ^{-1} \tau+\tau /\left(1+\tau^{2}\right)\right] / 4$. Omitting the details of the algebra, we here provide the resulting eddy viscosity $v_{\mathrm{T}}$, magnetic diffusivity $\eta_{\mathrm{T}}$ and heat diffusivity $\mu_{\mathrm{T}}$ :

$$
\begin{aligned}
\nu_{\mathrm{T}} & \simeq \frac{\tau_{\mathrm{f}}}{4 B_{0}^{2}} \int \frac{\mathrm{d}^{2} k}{(2 \pi)^{2}} \frac{\phi(\boldsymbol{k})}{k_{y}^{4}}\left[1-G\left(\bar{N}^{2}\right)\right] \sim \frac{1}{\omega_{\mathrm{A}}^{2}} \mathcal{F}, \\
\eta_{\mathrm{T}} & \simeq \frac{\tau_{\mathrm{f}}}{2 B_{0}^{2}} \int \frac{\mathrm{d}^{2} k}{(2 \pi)^{2}} \frac{\phi(\boldsymbol{k})}{k_{y}^{4}}\left(\frac{\xi_{\eta}}{3}\right)^{2 / 3} \Gamma\left(\frac{1}{3}\right) \mathrm{e}^{-\pi \bar{N}^{2} / 4} \lesssim \frac{\xi_{\eta}^{2 / 3}}{\omega_{\mathrm{A}}^{2}} \mathcal{F}, \\
\mu_{\mathrm{T}} & \simeq \frac{\tau_{\mathrm{f}}}{N^{2}} \int \frac{\mathrm{d}^{2} k}{(2 \pi)^{2}} \frac{\phi(\boldsymbol{k})}{k_{y}^{2}} \sim \frac{1}{N^{2}} \mathcal{F} .
\end{aligned}
$$

Here, $\omega_{\mathrm{A}}=\left|k_{y} B_{0}\right|, k_{y}$ is the typical wavenumber of the forcing, and $\mathcal{F} \sim \tau_{\mathrm{f}} \int \mathrm{d}^{2} k \phi(\boldsymbol{k}) / k_{y}^{2} \sim v_{\mathrm{f}}^{2} / \tau_{\mathrm{f}}$ is the strength of the forcing with a characteristic forcing velocity $v_{\mathrm{f}}$ and correlation time $\tau_{\mathrm{f}}$. The function $G\left(\bar{N}^{2}\right)$ depends on $\bar{N}^{2}$, taking at most a maximum value $\sim 0.37$ for $\bar{N}^{2} \sim 4$ and vanishing as either $\bar{N} \rightarrow 0$ or $\bar{N} \rightarrow \infty$. Since $G\left(\bar{N}^{2}\right)<1$, the eddy viscosity in Eq. (25) is now positive. That is, turbulent momentum transport in the limit of a strong radiative damping is diffusive, in contrast to the case of weak radiative damping considered in Sect. 3. This can be shown to be related to the equipartition between kinetic and magnetic energy to leading order, in contrast to the weakly damped case in Sect. 3. As expected, this is because the buoyancy force is greatly reduced by strong radiative damping. The results in Eqs. (25)-(27) show that both $\nu_{\mathrm{T}}$ and $\eta_{\mathrm{T}}$ are quenched largely by magnetic fields while $\eta_{\mathrm{T}}$ is also reduced by shear $\left(\propto \xi_{\eta}^{2 / 3} \propto \mathcal{A}^{-2 / 3}\right)$. In comparison, the heat diffusivity in Eq. (27) exhibits a different behavior, being mainly inhibited by buoyancy force. This is because the coupling of the density fluctuation to the fluid is feeble due to strong radiative damping.

The comparison of Eqs. (25) and (26) reveals that the magnetic diffusivity is smaller than eddy viscosity roughly by a factor of $\xi_{\eta}^{2 / 3} \ll 1$ (recall $\xi_{\eta}=\eta k_{y}^{2} / \mathcal{A}$ is a small parameter characterizing the strong shear limit, where $l_{\mathrm{f}}=1 / k_{y}$ is the characteristic scale of the forcing). The ratio $\eta_{\mathrm{T}} / \nu_{\mathrm{T}} \ll 1$ is however larger than the value in the case of weak damping in Sect. 3. This is an interesting result since this ratio is crucial in the estimate of the tachocline thickness, which has been taken to be large $\eta / v \sim 10^{2}$ by using the molecular values of $\eta$ and $v$ in previous works. That is, our predicted value $\eta_{\mathrm{T}} / \nu_{\mathrm{T}}(\ll 1)$ is much smaller than $\eta / v$ based on molecular values. Furthermore, the ratio of the effect of the hyperviscosity $\lambda_{\mathrm{T}}$ to that of eddy viscosity $\nu_{\mathrm{T}}$ is now roughly given by $\left(B_{0} / R_{\odot} N\right)^{2}\left(R_{\odot}{ }^{2} k_{y} / h\right)^{2}(\Omega / N)^{2} \gtrsim 1$ for reasonable parameter values. That is, the effect of hyperviscosity due to meridional circulation is crucial in maintaining the momentum balance in the tachocline.

Based on these observations, we now seek to obtain the estimate on the thickness of the tachocline and the strength $B_{y}$ and $B_{z}$ by requiring $\partial_{t}=0$ and by ignoring $v_{\mathrm{T}}$ compared to $\lambda_{\mathrm{T}} \sim \mu_{\mathrm{T}}(\Omega / N)^{2}\left(R_{\odot} / \Lambda\right)^{2}$ in Eq. (20). A simple analysis of Eqs. (20)-(21), with the help of Eqs. (26) and (27) and $U_{y} \sim$ $\mathcal{A} R_{\odot}$, gives us

$$
\begin{aligned}
\frac{h / R_{\odot}}{R_{\odot} k_{y}} & \sim \frac{1}{\Lambda}\left(\frac{\mathcal{A}}{N}\right)^{2} \xi_{\eta}^{-1 / 3}, \\
\frac{B_{z}}{B_{y}} & \sim \frac{\eta_{\mathrm{T}}}{\mathcal{A} h^{2}},
\end{aligned}
$$

where $\xi_{\eta}=\eta k_{y}^{2} / \mathcal{A}$ and $l_{\mathrm{f}}=1 / k_{y}$ is the characteristic length scale of the forcing. In a sharp contrast to the previous works which use the molecular values for the viscosity and magnetic diffusivity, the result (28) shows that the tachocline thickness is independent of the strength of magnetic fields. For instance, for $k_{y}=10^{-7}-10^{-6} \mathrm{~cm}^{-1}, h / R_{\odot}=0.01-0.05$ for $\mathcal{A} \sim 3 \times 10^{-6} \mathrm{~s}^{-1}$, $N \sim 3 \times 10^{-3} \mathrm{~s}^{-1}$, and $\Lambda \sim O(1)$, or $h / R_{\odot} \sim 10^{-3}$ for $N \sim 10^{-2} \mathrm{~s}^{-1}$. This estimate tends to be larger than $h / R_{\odot} \sim 10^{-3}$ by Gough \& McIntyre (1998), and is mainly due to the small magnetic diffusivity with the value $\eta_{\mathrm{T}} / \mu_{\mathrm{T}} \sim \xi_{\eta}^{2 / 3}\left(N / \omega_{\mathrm{A}}\right)^{2} \ll 1$. That is, a more efficient momentum transport than magnetic dissipation causes a thicker tachocline. Equation (28) implies that the tachocline thickness decreases as the stratification becomes stronger, which could have some implications for other stars. On the other hand, Eq. (29) shows that the ratio of magnetic field strengths depends on the tachocline thickness and the turbulent magnetic diffusivity $\eta_{\mathrm{T}}$. As $\eta_{\mathrm{T}}$ is directly proportional to the strength of the forcing $\mathcal{F}$, we again utilize the constraint on the turbulent particle diffusivity $D_{\mathrm{T}} \sim 10^{3} \mathrm{~cm}^{2} \mathrm{~s}^{-1}$ in the tachocline to be consistent with the surface depletion of lithium on the Sun. Since $\eta_{\mathrm{T}} \sim(\eta / D) D_{\mathrm{T}}\left(\right.$ see Kim 2006) where $D \sim 20 \mathrm{~cm}^{2} \mathrm{~s}^{-1}$ and $\eta \sim 10^{4} \mathrm{~cm}^{2} \mathrm{~s}^{-1}$, we obtain $\eta_{\mathrm{T}} \sim 10^{5} \mathrm{~cm}^{2} \mathrm{~s}^{-1}$. By using this value in Eq. (29) and $h / R_{\odot} \gtrsim 0.01-0.05$, we can obtain $B_{z} / B_{y} \sim 10^{-8}-10^{-7}$. If we take, $B_{y} \sim 10^{4} \mathrm{G}, B_{z} \sim 10^{-4}-10^{-3} \mathrm{G}$ while for $B_{y} \sim 10^{5} \mathrm{G}, B_{z} \sim 10^{-3}-10^{-2} \mathrm{G}$. These values of $B_{z}$ are comparable to the previously estimated value. It is important to note that for $\eta_{\mathrm{T}} \sim 10^{5} \mathrm{~cm}^{2} \mathrm{~s}^{-1}$, Eqs. (25) and (27) give $\nu_{\mathrm{T}} \sim 10^{6} \mathrm{~cm}^{2} \mathrm{~s}^{-1}$ and $\mu_{\mathrm{T}} \sim 10^{10} \mathrm{~cm}^{2} \mathrm{~s}^{-1}(\gg \mu)$. Therefore, our results naturally predict a more efficient momentum transport than particle transport (recall $D_{\mathrm{T}} \sim 10^{3} \mathrm{~cm}^{2} \mathrm{~s}^{-1}$ ) in the tachocline without fine-tuning parameters (see also Kim 2006), as required to be consistent with the observations.

\section{Discussion and conclusions}

We have presented a theory of turbulent transport in the sheared stratified magnetised tachocline when turbulence is driven and maintained by an external forcing (e.g. due to plumes penetrating from the convection zone). Despite the presence of a strong (uniform) magnetic field, the momentum transport is found to be anti-diffusive $\left(v_{\mathrm{T}}<0\right)$ for reasonable parameter values in the tachocline. The magnetic diffusivity $\left(\eta_{\mathrm{T}}\right)$ and heat diffusivity $\left(\mu_{\mathrm{T}}\right)$ (and thus hyperviscosity) are found to be positive, but severely reduced as a result of the shear stabilization and transport reduction by Alfven-gravity waves with very small values of $\eta_{\mathrm{T}} /\left|v_{\mathrm{T}}\right|(\ll 1)$ and $\mu_{\mathrm{T}} /\left|\nu_{\mathrm{T}}\right|(\ll 1)$. Since the momentum transport is anti-diffusive, the gradient in the radial differential rotation is amplified. As the shear becomes strong, the hyperviscosity due to the meridional circulation, which is always diffusive, can become important, counteracting the effect of negative eddy viscosity. However, in order to maintain the radial shear $\mathcal{A}$ in the differential rotation with a value comparable to the average solar rotation $\Omega$, as presently inferred, the tachocline as the Hartmann layer has to be very thin with the thickness $h / R_{\odot} \sim 10^{-5}$ for the 
toroidal magnetic field $B_{y} \sim 10^{5} \mathrm{G}$. Here, $R_{\odot}$ is the solar radius. Otherwise, the radial shear and/or toroidal magnetic field in the tachocline appears to be too strong to be stable against the shear instability and/or magnetic buoyancy instability. Furthermore, since the turbulent coefficients depend nonlinearly on $\mathcal{A}$ and $B_{y}$, $\mathcal{A}$ and $B_{y}$ form a nonlinear dynamical system, which can exhibit complex time variation. These results point to the possibility that the tachocline may exhibit a much more complex (temporal and/or spatial dependent) dynamics than previously thought even in the slow tachocline scenario.

In the limit of strong radiative damping where the temperature (density) fluctuation is almost stationary, the turbulent momentum transport is found to be diffusive, down the gradient. This requires the turbulence to be on very small scales such that $\xi_{\mu}=\mu k_{y}^{2} / \mathcal{A} \gg 1$. Here, $1 / k_{y}=l_{\mathrm{f}}$ is the characteristic length scale of the forcing, and $\mathcal{A} \sim 3 \times 10^{-6} \mathrm{~s}^{-1}$ is the radial shear. Thus, the forcing scale $l_{\mathrm{f}}$ has to be smaller than $10^{6}-10^{7} \mathrm{~cm}$, which is about $10^{-4} R_{\odot}$. In this case, the tachocline may be viewed as a Hartmann layer with possibly stable configurations of the radial differential rotation and toroidal magnetic field. A simple analysis by using the predicted values of $\nu_{\mathrm{T}}, \mu_{\mathrm{T}}$, and $\eta_{\mathrm{T}}$ suggested that the effect of the hyperviscosity is likely to dominate over the eddy viscosity. However, since the magnetic diffusion is severely quenched compared with heat diffusivity, the momentum transport is effectively much more efficient than the magnetic field diffusion. Consequently, the major force balance in the tachocline leads to the estimate of the tachocline thickness $\left(\gtrsim 10^{-2} R_{\odot}\right)$ for $N \sim 3 \times 10^{-3} \mathrm{~s}^{-1}$, which tends to be larger than the previously estimated value $\sim 10^{-3} R_{\odot}$ by Gough \& McIntyre (1998). As $\eta_{\mathrm{T}}$ and $\mu_{\mathrm{T}}$ depend on the strength of the toroidal magnetic fields and radial shear, the tachocline thickness is found to be independent of the strength of (toroidal and/or poloidal) magnetic field (cf. Gough \& McIntyre 1998). The strength of poloidal magnetic field in the radiative interior is estimated to be of order $10^{-4}-10^{-2} \mathrm{G}$ for a value of the toroidal magnetic field $10^{4}-10^{5} \mathrm{G}$, comparable to the previously estimated value. Importantly, the results provide a natural explanation for a more efficient momentum transport than particle mixing in the tachocline. However, a severe reduction in the magnetic field diffusion could be problematic for the solar dynamo (e.g., the interface dynamo, Parker 1993).

While the discussion of our results is focused on the applications to the present sun, they might also have interesting implications for other stars. In particular, our predicted values of turbulent transport coefficients have different dependences on the strength of magnetic field, stratification, and radial shear as well as on the molecular values of viscosity, ohmic diffusivity, and radiative diffusivity. As the values of these parameters vary from one star to another, it would be of interest to explore the implications of these results for other stars. For example, it might be possible to utilise the results to infer the value of the radial shear or the strength of magnetic field in other stars, and also to gain some insight into the presence of a tachocline-like shear layer in those. Of course, in the case of more massive stars, the rotation rate is much faster than that of the sun, demanding the prediction of turbulent transport coefficients by taking into account the effect of average rotation (Leprovost \& Kim 2007). Ultimately, it will be interesting to investigate a consistent model incorporating the spin-down of the sun (and other stars) due to the angular momentum loss. Finally, we note that our analysis, limited to 2D, should be extended to 3D, in particular, to study solar dynamos (e.g. the $\alpha$ effect). These issues are currently under investigation and will be addressed in future publications.

Acknowledgements. This work was supported by the PPARC Grant $\mathrm{PP} / \mathrm{B} 501512 / 1$.

\section{References}

Barnes, G., Charbonneau, P., \& MacGregor, K. B. 1999, ApJ, 511, 466

Cattaneo, F., \& Vainshtein, S. I. 1991, ApJ, 376, L21

Charbonnel, C., \& Talon, S. 2005, Science, 309, 2189

Drazin, P. G., Reid, W. H. 1981, Hydrodynamic stability (Cambridge: Cambridge University Press), 327

Gilman, P. A. 2000, Sol. Phys., 192, 27

Gough, D. O., \& McIntyre, M. E. 1998, Nature, 394, 755

Gruzinov, A. V., \& Diamond, P. H. 1994, Phys. Rev. Lett., 72, 1651

Kim, E. 2004, Mod. Phys. Lett. B, 18, 1

Kim, E. 2005, A\&A, 441, 763

Kim, E. 2006, Phys. Rev. Lett., 96, 084504

Kim, E., \& Dubrulle, B. 2001, Phys. Plasmas, 8, 813

Kim, E., \& Leprovost, N. 2007, A\&A, submitted

Kim, E., \& MacGregor, K. B. 2001, ApJ, 556, L117

Leprovost, N., \& Kim, E. 2006, A\&A, 456, 617

Leprovost, N., \& Kim, E. 2007, A\&A, 463, L9

MacGregor, K. B., \& Charbonneauu, P. 1999, ApJ, 519, 911

Parker, E. N. 1993, ApJ, 408, 707

Petrovay, K. 2003, Sol. Phys., 215, 17

Pinsonneault, M., Kawaler, S. D., Sofia, S., \& Demarque, P. 1989, ApJ, 338, 424

Rüdiger, G., \& Kitchatinov, L. L. 1996, ApJ, 466, 1078

Schatzman, F. D. 1993, The stars (Berlin, London: Springer-Verlag)

Spiegel, E. A., \& Zahn, J.-P. 1992, A\&A, 265, 106

Spruit, H. C. 1999, A\&A, 349, 189

Stix, M. 1989, The sun: an introduction (Berlin, London: Springer-Verlag) 